\title{
UPPER TRIANGULAR TECHNOLOGY AND THE ARF-KERVAIRE INVARIANT
}

\author{
VICTOR P. SNAITH
}

\begin{abstract}
This paper introduces the upper triangular technology (UTT) into classical homotopy theory. This is a new and easy to use method to calculate the effect of the left unit map in 2-adic connective K-theory; the map which is the basis for operations in bu-theory. By way of application, UTT is used to give a new, very simple proof of a conjecture of BarrattJones-Mahowald, which rephrases K-theoretically the existence of framed manifolds of Arf-Kervaire invariant one.
\end{abstract}

\section{INTRODUCTION}

1.1. The upper triangular technology (UTT) referred to in the title consists of the following two results. Let bu and bo denote the stable homotopy spectra representing 2-adically completed unitary and orthogonal connective K-theory respectively. Thus the smash product, $b u \wedge b o$ is a left $b u$-module spectrum and so we may consider the ring of left $b u$-module endomorphisms of degree zero in the stable homotopy category of spectra [2], which we shall denote by End left-bu-mod$(b u \wedge b o)$. The group of units in this ring will be denoted by $A u t_{l e f t-b u-m o d}(b u \wedge b o)$, the group of homotopy classes of left $b u$-module homotopy equvialences and let $A u t_{l e f t-b u-m o d}^{0}(b u \wedge b o)$ denote the subgroup of left $b u$-module homotopy equivalences which induce the identity map on $H_{*}(b u \wedge b o ; \mathbb{Z} / 2)$.

Let $U_{\infty} \mathbb{Z}_{2}$ denote the group of infinite, invertible upper triangular matrices with entries in the 2-adic integers. That is, $X=\left(X_{i, j}\right) \in U_{\infty} \mathbb{Z}_{2}$ if $X_{i, j} \in \mathbb{Z}_{2}$ for each pair of integers $0 \leq i, j$ and $X_{i, j}=0$ if $j<i$ and $X_{i, i}$ is a 2-adic unit.

Theorem 1.2. ([20] §2.1)

There is an isomorphism of the form

$$
\Psi: A u t_{l e f t-b u-\text { mod }}^{0}(b u \wedge b o) \stackrel{\cong}{\longrightarrow} U_{\infty} \mathbb{Z}_{2} .
$$

Let $\psi^{3}:$ bo $\longrightarrow$ bo denote the Adams operation.

This isomorphism $\Psi$ is defined up to inner automorphisms of $U_{\infty} \mathbb{Z}_{2}$. Given an important automorphism in $\mathrm{Aut}_{\text {left-bu-mod }}^{0}(b u \wedge b o)$ one is led to ask what is its conjugacy class in $U_{\infty} \mathbb{Z}_{2}$. By far the most important such automorphism is $1 \wedge \psi^{3}$.

Date: 26 September 2006. 
Theorem 1.3. ([10] §1.1)

Under the isomorphism $\Psi$ the automorphism $1 \wedge \psi^{3}$ corresponds to an element in the conjugacy class of the matrix

$$
\left(\begin{array}{cccccc}
1 & 1 & 0 & 0 & 0 & \ldots \\
0 & 9 & 1 & 0 & 0 & \ldots \\
0 & 0 & 9^{2} & 1 & 0 & \ldots \\
0 & 0 & 0 & 9^{3} & 1 & \ldots \\
\vdots & \vdots & \vdots & \vdots & \vdots & \vdots
\end{array}\right)
$$

To be precise, the proof in ([10] §1.1) shows that $1 \wedge \psi^{3}$ can be conjugated to this form in its first $N$ columns for arbitarily large $N$. In Example 3.2 I shall explain what this means in practice.

The main purpose of this paper is to illustrate UTT at work in an application. By way of illustration I shall use UTT to give an elementary proof of the following result, whose terminology and proof will be given in $\S 3$.

\section{Theorem 1.4.}

Let $m$ be a positive integer and let $\Theta_{8 m-2}: S^{8 m-2} \longrightarrow \mathbb{R P}^{8 m-2}$ be a morphism in the 2-local stable homotopy category. Then the bo e-invariant of $\Theta_{8 m-2}$ is $\left(3^{4 m}-1\right) / 4$ (modulo $2^{4 m-1}$ ) if and only if $m=2^{q}$ and $\Theta_{8 m-2}$ is detected by the Steenrod operation $S q^{q^{2+2}}$.

Any 2-adic stable homotopy class $\tilde{\Theta} \in \pi_{8 m-2}\left(S^{0}\right) \otimes \mathbb{Z}_{2}$ lifts canonically (via the Kahn-Priddy Theorem [3] [12]) to an element of the stable homotopy of $\mathbb{R} \mathbb{P}^{\infty}$ and thence to $\Theta_{8 m-2}$. Detection by a primary mod 2 cohomology operation can only occur if $m$ is a power of 2 and is equivalent to $\tilde{\Theta}$ being represented by a framed manifold of Arf-Kervaire invariant one ([8], [11], [18], [21]). The existence or otherwise of framed manifolds of Arf-Kervaire invariant one is a classical unsolved problem in homotopy theory. As explained in [13], the alternative formulation of Theorem 1.1 is equivalent to a conjecture of [7] which was first proved in [14] by a very difficult study of Ad-theory and is proved in [21] by a straightforward but brutally long-winded use of BP-operations. The attempted proof of [13] contains a gap caused by lack of control of the filtration in an Adams spectral sequence. Intuitively, the UTT proof of Theorem 1.1 is conceptually simple because it amounts to inflicting the relevant mod 2 Adams spectral sequence with a "mixed Hodge structure"; that is, a direct sum decomposition (corresponding to the entries in $U_{\infty} \mathbb{Z}_{2}$ ) compatible with the usual Adams filtration.

Incidentally Theorem 1.4 poses the existence of framed manifolds of ArfKervaire invariant one in a form which is similar to the formulation of the existence of framed manifolds of Hopf invariant one in terms of 
$\Theta_{2 m-1}: S^{2 m-1} \longrightarrow \mathbb{R P}^{2 m-1}$ (see [19]). These only exist for dimensions $1,3,7$ and currently framed manifolds of Arf-Kervaire invariant one have been constructed in dimensions 2, 6, 14,30,62 (see [18] and [15] - I believe that [16] has a gap in its construction). Accordingly the following conjecture seems reasonable:

\section{Conjecture 1.5.}

Framed manifolds of Arf-Kervaire invariant one exist at most in dimensions $2,6,14,30$ and 62 .

The paper is organised in the following manner. The basis of Theorems 1.2 and 1.3 is the left $b u$-module splitting of $b u \wedge b o$ into a sum of spectra of the form $b u \wedge\left(F_{4 k} / F_{4 k-1}\right)$ for $k \geq 0$. In $\S 2$ the mod 2 Adams spectral sequence for the homotopy of $b u \wedge\left(F_{4 k} / F_{4 k-1}\right) \wedge \mathbb{R P}^{2 m}$ is described. The crucial result is the multiplicative structure stated in Theorem 2.6 and proved in $\S 2.8$. Combined with results from [10], Theorem 2.6 yields Proposition 2.10, which evaluates the effect on homotopy of the maps corresponding to the superdiagonal entries of an upper-triangular matrix. These maps correspond to the 1 's in the matrix for $1 \wedge \psi^{3}$ in Theorem 1.3. In $\S 3$ Theorem 1.3 is combined with Proposition 2.10 to transform expressions for the bo e-invariant of $\Theta_{8 m-2}$ into a series of 2-adic equations in Example 3.4 (and Propositions 3.5 and 3.6) from which Theorem 1.4 is easily deduced in $\S 3.8$.

I am very grateful for help and advice to Huajian Yang, my postdoc at McMaster University in 1996-98, to Jonathan Barker, my PhD student at the University of Southampton in 2003-2006 and to Francis Clarke.

\section{K-theory examples}

2.1. Let $b u_{*}(X)$ (resp. $K U_{*}(X)$ ) denote the reduced, connective (resp. periodic) complex K-theory of a (based) CW complex $X$. When $X$ equals the zero-dimensional sphere we have $b u_{*}\left(S^{0}\right) \cong \mathbb{Z}[u]$ and $K U_{*}\left(S^{0}\right) \cong \mathbb{Z}\left[u^{ \pm 1}\right]$ where $\operatorname{deg}(u)=2$. Let $\mathbb{R P}^{n}$ denote $n$-dimensional real projective space. Let $\mathbb{Z} / t\langle w\rangle$ denote a cyclic group of order $t$ with generator $w$ The following result is well known.

Proposition 2.2. For $1 \leq m \leq \infty$

$$
b u_{j}\left(\mathbb{R P}^{2 m}\right)= \begin{cases}0 & \text { if } j \text { is even, } \\ \mathbb{Z} / 2^{i}\left\langle v_{2 i-1}\right\rangle & \text { if } 1 \leq j=2 i-1<2 m, \\ \mathbb{Z} / 2^{m}\left\langle v_{2 m-1} u^{i-m}\right\rangle & \text { if } 2 m<j=2 i-1 .\end{cases}
$$

In addition, the generators may be chosen to satisfy $u v_{2 i-1}=2 v_{2 i+1}$ for $1 \leq$ $i \leq m-1$. 


\section{Proof}

The Atiyah-Hirzebruch spectral sequences for computing $b u_{*}\left(\mathbb{R P}^{2 m}\right)$ and $K U_{*}\left(\mathbb{R} \mathbb{P}^{2 m}\right)$ collapse for dimensional reasons. This implies that $b u_{j}\left(\mathbb{R} \mathbb{P}^{2 m}\right)$ has the correct order. It also implies the injectivity of the canonical maps

$$
h_{j}\left(\mathbb{R} \mathbb{P}^{2 m}\right) \longrightarrow h_{j}\left(\mathbb{R} \mathbb{P}^{2 m+2}\right)(h=b u, K U), \lambda_{*}: b u_{j}\left(\mathbb{R} \mathbb{P}^{2 m}\right) \longrightarrow K U_{j}\left(\mathbb{R} \mathbb{P}^{2 m}\right) \text {. }
$$

However, by the universal coefficient theorem for $K U$ and the results of ([5] p.107) we have $K U_{2 i-1}\left(\mathbb{R} P^{\infty}\right) \cong \mathbb{Z} / 2^{\infty}$ so each $b u_{2 i-1}\left(\mathbb{R P} P^{2 m}\right)$ is cyclic. The relation $u v_{2 i-1}=2 v_{2 i+1}$ follows from Bott periodicity and the fact that the injection $\lambda_{*}$ commutes with multiplication by $u$.

2.3. $\operatorname{Ext}_{B}^{*, *}\left(\tilde{H}^{*}\left(\mathbb{R P}^{2 m} ; \mathbb{Z} / 2\right), \mathbb{Z} / 2\right)$

Let $B=E\left(S q^{1}, S q^{0,1}\right)$ denote the exterior subalgebra of the mod 2 Steenrod algebra $\mathcal{A}[22]$ generated by $S q^{1}$ and $S q^{0,1}=\left[S q^{1}, S q^{0,1}\right]$. There is an isomorphism of bigraded algebras $\operatorname{Ext}_{B}^{*, *}(\mathbb{Z} / 2, \mathbb{Z} / 2) \cong \mathbb{Z} / 2[a, b]$, the polynomial algebra on $a$ and $b$ with $\operatorname{bideg}(a)=(1,1), \operatorname{bideg}(b)=(1,3)$. Also $\tilde{H}^{*}\left(\mathbb{R P}^{2 m} ; \mathbb{Z} / 2\right)=\left\langle x, x^{2}, \ldots\right\rangle /\left(x^{2 m+1}\right)$ with $S q^{1}\left(x^{n}\right)=n x^{n+1}, S q^{0,1}\left(x^{n}\right)=$ $n x^{n+3}$.

Consider the bigraded $\mathbb{Z} / 2[a, b]$-module $\operatorname{Ext}_{B}^{* * *}\left(\tilde{H}^{*}\left(\mathbb{R P}^{2 m} ; \mathbb{Z} / 2\right), \mathbb{Z} / 2\right)$. Denote the non-zero element of $\operatorname{Ext}_{B}^{0,2 i-1}\left(\tilde{H}^{*}\left(\mathbb{R} \mathbb{P}^{2 m} ; \mathbb{Z} / 2\right), \mathbb{Z} / 2\right)$ by $\tilde{v}_{2 i-1}$ for $1 \leq$ $i \leq m$.

Proposition 2.4. For $1 \leq m \leq \infty$ the bigraded $\operatorname{Ext}_{B}^{*, *}(\mathbb{Z} / 2, \mathbb{Z} / 2)$-module $\operatorname{Ext}_{B}^{*, *}\left(\tilde{H}^{*}\left(\mathbb{R} \mathbb{P}^{2 m} ; \mathbb{Z} / 2\right), \mathbb{Z} / 2\right)$ is equal to

$$
\frac{\mathbb{Z} / 2[a, b]\left\langle\tilde{v}_{1}, \tilde{v}_{3}, \ldots, \tilde{v}_{2 m-1}\right\rangle}{\left\{a^{i} \tilde{v}_{2 i-1}, b \tilde{v}_{2 i-1}-a \tilde{v}_{2 i+1}\right\}}
$$

\section{Proof}

We prove this by induction on $m$. When $m=1$ we have

$$
\operatorname{Ext}_{B}^{*, *}\left(\tilde{H}^{*}\left(\mathbb{R P}^{2} ; \mathbb{Z} / 2\right), \mathbb{Z} / 2\right) \cong \operatorname{Ext}_{B}^{* * *}\left(E\left(S q^{1}\right)[1], \mathbb{Z} / 2\right) \cong \mathbb{Z} / 2[b]\left\langle\tilde{v}_{1}\right\rangle
$$

where $X[n]$ denotes $X$ with a dimension shift by $n$ so that $X[1]=\Sigma X$ in the notation of [2] and [20]. We have a short exact sequence of $B$-modules

$$
0 \longrightarrow \tilde{H}^{*}\left(\mathbb{R} \mathbb{P}^{2 m-2} ; \mathbb{Z} / 2\right)[-2] \stackrel{\left(x^{2} \cdot-\right)}{\longrightarrow} \tilde{H}^{*}\left(\mathbb{R} \mathbb{P}^{2 m} ; \mathbb{Z} / 2\right) \longrightarrow \tilde{H}^{*}\left(\mathbb{R} \mathbb{P}^{2} ; \mathbb{Z} / 2\right) \longrightarrow 0 \text {. }
$$

By induction, for each non-negative integer $r$ the resulting long exact sequence yields an upper bound for the sums of $\mathbb{F}_{2^{-}}$dimensions

$$
\sum_{s=0}^{\infty} \sum_{t-s=r} \operatorname{dim}_{\mathbb{F}_{2}}\left(\operatorname{Ext}_{B}^{s, t}\left(\tilde{H}^{*}\left(\mathbb{R P}^{2 m} ; \mathbb{Z} / 2\right), \mathbb{Z} / 2\right)\right) \leq d_{r}
$$

where $d_{r}=0$ if $r$ is even, $d_{2 i-1}=i$ for $1 \leq i \leq m$ and $d_{2 i-1}=m$ for $m \leq i$. On the other hand, if $\mathbb{Z}_{2}$ denotes the 2-adic integers, the Adams spectral sequence $([2])$ for $\pi_{*}\left(b u \wedge \mathbb{R P}^{2 m}\right) \otimes \mathbb{Z}_{2}=b u_{*}\left(\mathbb{R P}^{2 m}\right) \otimes \mathbb{Z}_{2}$ has the form $([10]$; [20])

$$
E_{2}^{s, t}=\operatorname{Ext}_{B}^{s, t}\left(\tilde{H}^{*}\left(\mathbb{R P}^{2 m} ; \mathbb{Z} / 2\right), \mathbb{Z} / 2\right) \Longrightarrow b u_{t-s}\left(\mathbb{R P}^{2 m}\right) \otimes \mathbb{Z}_{2}
$$


and collapses for dimensional reasons, being concentrated where $t-s$ is odd. Therefore Proposition 2.2 shows that $d_{r}$ is also a lower bound. The relations folllow from the fact that $a$ and $b$ represent 2 and $u$ respectively in the Adams spectral sequence for $b u_{*}\left(S^{0}\right) \otimes \mathbb{Z}_{2}$.

\section{5. $\operatorname{Ext}_{B}^{*, *}\left(\tilde{H}^{*}\left(X \wedge\left(F_{4 k} / F_{4 k-1}\right) ; \mathbb{Z} / 2\right), \mathbb{Z} / 2\right)$}

Consider the second loopspace of the 3 -sphere, $\Omega^{2} S^{3}$. There exists a model for $\Omega^{2} S^{3}$ which is filtered by finite complexes $([9],[17])$

$$
S^{1}=F_{1} \subset F_{2} \subset F_{3} \subset \ldots \subset \Omega^{2} S^{3}=\bigcup_{k \geq 1} F_{k}
$$

and there is a stable homotopy equivalence, an example of the so-called Snaith splitting, of the form $\Omega^{2} S^{3} \simeq \vee_{k \geq 1} F_{k} / F_{k-1}$.

Consider the finite complexes $F_{4 k} / F_{4 k-1}$ with the convention that $F_{0} / F_{-1}=$ $S^{0}$, the 0 -sphere. Let $\alpha(n)$ denote the number of 1's in the dyadic expansion of the positive integer $n$. The results of Adams-Margolis ([2], [4]; see also [10] and [20]) yield $\operatorname{Ext}_{B}^{* * *}(\mathbb{Z} / 2, \mathbb{Z} / 2)$-module isomorphisms of the form

$$
\begin{aligned}
& \operatorname{Ext}_{B}^{s, t}\left(\tilde{H}^{*}\left(X \wedge\left(F_{4 k} / F_{4 k-1}\right) ; \mathbb{Z} / 2\right), \mathbb{Z} / 2\right) \\
& \cong \operatorname{Ext}_{B}^{s+2 k-\alpha(k), t-2 k-\alpha(k)}\left(\tilde{H}^{*}(X ; \mathbb{Z} / 2), \mathbb{Z} / 2\right)
\end{aligned}
$$

for all $s>0$. We shall need this isomorphism in the case where $X$ is either a real projective space or a sphere. The case when $X$ is a sphere is described extensively in [20] in connection with the left bu-module equivalence of 2-local spectra (see also $([10] \S 2)$

$$
\hat{L}: \vee_{k \geq 0} \text { bu } \wedge\left(F_{4 k} / F_{4 k-1}\right) \stackrel{\simeq}{\longrightarrow} b u \wedge \text { bo. }
$$

The groups $\operatorname{Ext}_{B}^{s, t}\left(\tilde{H}^{*}\left(\mathbb{R P}^{2 m} \wedge\left(F_{4 k} / F_{4 k-1}\right) ; \mathbb{Z} / 2\right), \mathbb{Z} / 2\right)$, when depicted in the traditional Adams spectral sequence manner with $s$ along the vertical and $t-s$ along the horizontal axis, looks as in the figure below. The figure is interpreted as follows: the groups are $\mathbb{F}_{2}$-vector spaces which are possibly non-zero only when $s=0$ and $t-s \geq 4 k+1$ or a copy of $\mathbb{Z} / 2$ at each point with $(s, t-s)=(v, 8 k-2 \alpha(k)-1+2 w+2 v)$ with $v=1,2,3, \ldots$ and $1 \leq w \leq m$. 


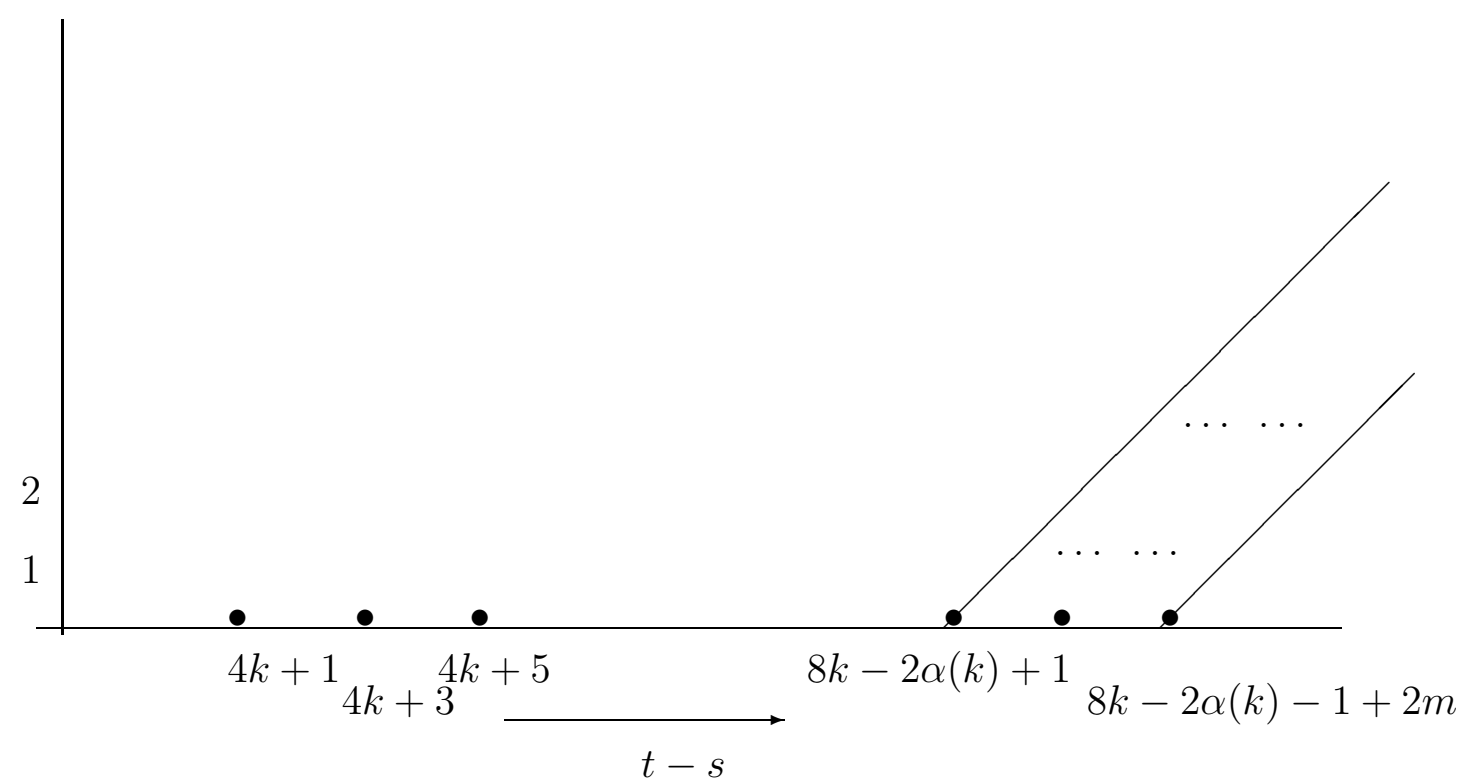

We have the following result describes the important aspects of the module structure over the bigraded algebra $\operatorname{Ext}_{B}^{*, *}(\mathbb{Z} / 2, \mathbb{Z} / 2) \cong \mathbb{Z} / 2[a, b]$. Let $\tilde{v}_{2 i-1} \in \operatorname{Ext}_{B}^{0,2 i-1}\left(\tilde{H}^{*}\left(\mathbb{R P}^{2 m} ; \mathbb{Z} / 2\right), \mathbb{Z} / 2\right)$ be as in Proposition 2.4 and let $\hat{z}_{4 k} \in$ $\operatorname{Ext}_{B}^{0,4 k}\left(\tilde{H}^{*}\left(F_{4 k} / F_{4 k-1} ; \mathbb{Z} / 2\right), \mathbb{Z} / 2\right)$ be element represented as a homomorphism on mod 2 cohomology by the inclusion of the bottom cell of $F_{4 k} / F_{4 k-1}$ ([10] Theorem 2.12). Hence we have a (non-zero) external product $\tilde{v}_{2 i-1} \hat{z}_{4 k} \in$ $\operatorname{Ext}_{B}^{0,4 k+2 i-1}\left(\tilde{H}^{*}\left(\mathbb{R P}^{2 m} \wedge\left(F_{4 k} / F_{4 k-1}\right) ; \mathbb{Z} / 2\right), \mathbb{Z} / 2\right)$ for $1 \leq i \leq m$. 


\section{Theorem 2.6.}

In $\operatorname{Ext}_{B}^{s, t}\left(\tilde{H}^{*}\left(\mathbb{R P}^{2 m} \wedge\left(F_{4 k} / F_{4 k-1}\right) ; \mathbb{Z} / 2\right), \mathbb{Z} / 2\right)$ we have:

(i) $\tilde{v}_{i} \hat{z}_{4 k} \neq 0$ for each $1 \leq i \leq m$,

(ii) $b \tilde{v}_{i} \hat{z}_{4 k}=0=a \tilde{v}_{i} \hat{z}_{4 k}$ for $i=1, \ldots, 2 k-\alpha(k)$,

(iii) $b^{e} \tilde{v}_{i} \hat{z}_{4 k} \neq 0$ for $e \geq 1$ and $i=2 k-\alpha(k)+1, \ldots, m$.

The proof of Theorem 2.6 will be given in $\S 2.8$ after some preliminaries concerning $B$-resolutions.

\subsection{Resolutions}

For $1 \leq m \leq \infty$, the $B$-action on $\tilde{H}^{*}\left(\mathbb{R} \mathbb{P}^{2 m} ; \mathbb{Z} / 2\right)=\left\langle x, x^{2}, \ldots, x^{2 m}\right\rangle$ is given by $S q^{1}\left(x^{i}\right)=i x^{i+1}$ and $S q^{0,1}\left(x^{i}\right)=i x^{i+3}$. The beginning of a free $B$-resolution

$$
\ldots \stackrel{d}{\longrightarrow} P_{1} \stackrel{d}{\longrightarrow} P_{0} \stackrel{\epsilon}{\longrightarrow} \tilde{H}^{*}\left(\mathbb{R} P^{2 m} ; \mathbb{Z} / 2\right) \longrightarrow 0
$$

may be given by $P_{0}=B\left\langle\sigma_{1}, \sigma_{3}, \ldots, \sigma_{2 m-1}\right\rangle$ and $P_{1}=B\left\langle\nu_{5}, \nu_{4}, \nu_{6}, \ldots, \nu_{2 m}\right\rangle$ where $\operatorname{deg}\left(\sigma_{i}\right)=i, \epsilon\left(\sigma_{i}\right)=x^{i}$ and $d\left(\nu_{2 t}\right)=S q^{1} \sigma_{2 t-1}+S q^{0,1} \sigma_{2 t-3}$ and $d\left(\nu_{5}\right)=$ $S q^{1} S q^{0,1} \sigma_{1}$. By Proposition $2.4 b \tilde{v}_{2 i-1}$ is the only non-zero element in the group $\operatorname{Ext}_{B}^{1,2 i+2}\left(\tilde{H}^{*}\left(\mathbb{R} \mathbb{P}^{2 m} ; \mathbb{Z} / 2\right), \mathbb{Z} / 2\right)$ for $1 \leq i \leq m$. Therefore it must be represented by homomorphism $h_{i} \in \operatorname{Hom}_{B}\left(P_{1}, \mathbb{Z} / 2\right)$ given by $h_{i}\left(\nu_{2 i+2}\right) \equiv 1$ (modulo 2) and $\mathrm{h}_{i}\left(\nu_{j}\right) \equiv 0$ otherwise.

Let $H(k)$ be the graded $\mathbb{F}_{2}$-vector space with basis

$$
y_{k, 2^{k}}, y_{k, 2^{k}+2}, y_{k, 2^{k}+4}, \ldots, y_{k, 2^{k+1}-2}, y_{k, 2^{k}+3}, y_{k, 2^{k}+5}, \ldots, y_{k, 2^{k+1}-1}
$$

where $\operatorname{deg}\left(y_{i}\right)=i$, with the "lightning flash" $B$-module structure given by

$$
S q^{0,1} y_{k, 2^{k}}=y_{k, 2^{k}+3}=S q^{1} y_{k, 2^{k}+2}, \ldots, S q^{0,1} y_{k, 2^{k+1}-4}=y_{k, 2^{k+1}-1}=S q^{1} y_{k, 2^{k+1}-2} \text {. }
$$

We define the start of a free $B$-resolution

$$
\ldots \longrightarrow R_{k, 1} \stackrel{d(k)}{\longrightarrow} R_{k, 0} \stackrel{\epsilon(k)}{\longrightarrow} H(k) \longrightarrow 0
$$

by $R_{k, 0}=B\left\langle\Sigma_{k, 0,2^{k}}, \Sigma_{k, 0,2^{k}+2}, \ldots, \Sigma_{k, 0,2^{k+1}-2}\right\rangle$ where $\operatorname{deg}\left(\Sigma_{k, 0, i}\right)=i$ and $R_{k, 1}=B\left\langle\Sigma_{k, 1,2^{k}+1}, \Sigma_{k, 1,2^{k}+3}, \ldots, \Sigma_{k, 1,2^{k+1}+1}\right\rangle$ where $\operatorname{deg}\left(\Sigma_{k, 1, i}\right)=i$. Also $\epsilon(k)$ and $d(k)$ are given by $\epsilon(k)\left(\Sigma_{k, 0,2 i}\right)=y_{k, 2 i}$ and

$$
\begin{aligned}
& d(k)\left(\Sigma_{k, 1,2^{k}+1}\right)=S q^{1} \Sigma_{k, 0,2^{k}}, \\
& d(k)\left(\Sigma_{k, 1,2^{k}+3}\right)=S q^{1} \Sigma_{k, 0,2^{k}+2}+S q^{0,1} \Sigma_{k, 0,2^{k}}, \\
& d(k)\left(\Sigma_{k, 1,2^{k}+5}\right)=S q^{1} \Sigma_{k, 0,2^{k}+4}+S q^{0,1} \Sigma_{k, 0,2^{k}+2}, \\
& \vdots \quad \vdots \quad \vdots \\
& d(k)\left(\Sigma_{k, 1,2^{k+1}+1}\right)=S q^{0,1} \Sigma_{k, 0,2^{k+1}-2} .
\end{aligned}
$$

We are now ready to embark on the proof of Theorem 2.6. 


\subsection{Proof of Theorem 2.6}

Part (i) follows since the exterior product of two non-zero $B$-homomorphisms to $\mathbb{Z} / 2$ is also non-zero and part (ii) follows because the elements in question lie in groups which are zero, by Proposition 2.4 and the discussion of $§ 2.5$. Part (iii) is more substantial. By naturality it suffices to work with $m=\infty$.

Let $k=2^{\epsilon_{1}}+2^{\epsilon_{2}}+\ldots+2^{\epsilon_{t}}$ with $0 \leq \epsilon_{1}<\epsilon_{2}<\ldots<\epsilon_{t}$ so we are interested in $i \geq 2^{\epsilon_{1}+1}+2^{\epsilon_{2}+1}+\ldots+2^{\epsilon_{t}+1}-t+1$ and $4 k=2^{\epsilon_{1}+2}+2^{\epsilon_{2}+2}+\ldots+2^{\epsilon_{t}+2}$. From ([2] pp.341-2) or ([20] p.1267) $\tilde{H}^{*}\left(F_{4 k} / F_{4 k-1} ; \mathbb{Z} / 2\right) \cong \otimes_{j=1}^{t} H\left(\epsilon_{j}+2\right)$. We have a free $B$-resolution given by the tensor product

$$
\ldots \rightarrow \otimes_{j=1, \sum_{a_{j}=1}^{t}} R_{\epsilon_{j}+2, a_{j}} \stackrel{d}{\rightarrow} \otimes_{j=1}^{t} R_{\epsilon_{j}+2,0} \rightarrow \tilde{H}^{*}\left(F_{4 k} / F_{4 k-1} ; \mathbb{Z} / 2\right) \rightarrow 0 .
$$

We introduce the convention that

$$
\Sigma_{\epsilon_{j}+2,1,2 s+1}=0=\Sigma_{\epsilon_{j}+2,0,2 s} \text { if } s \leq 2^{\epsilon_{j}+1} \text { or } 2^{\epsilon_{j}+2} \leq s .
$$

With this convention the differential has the form

$$
d\left(\epsilon_{j}+2\right)\left(\Sigma_{\epsilon_{j}+2,1,2 s+1}\right)=S q^{1} \Sigma_{\epsilon_{j}+2,1,2 s}+S q^{0,1} \Sigma_{\epsilon_{j}+2,1,2 s-2} .
$$

The element $\hat{z}_{4 k}$ is represented by the $B$-homomorphism

$$
g_{k} \in \operatorname{Hom}_{B}\left(\otimes_{j=1}^{t} R_{\epsilon_{j}+2,0}, \mathbb{Z} / 2\right)
$$

given by $g_{k}\left(\otimes_{j=1}^{t} \Sigma_{\epsilon_{j}+2,0,2^{\epsilon_{j}+2}}\right) \equiv 1$ (modulo 2) and $g_{k}\left(\otimes_{j=1}^{t} \Sigma_{\epsilon_{j}+2,0, w_{j}}\right) \equiv 0$ otherwise.

We must show that there does not exist a $B$-homomorphism

$$
f \in \operatorname{Hom}_{B}\left(P_{0} \otimes\left(\otimes_{j=1}^{t} R_{\epsilon_{j}+2,0}\right), \mathbb{Z} / 2\right)
$$

such that $f \cdot d=\left(0, h_{i} \otimes g_{k}\right)$ in the group of $B$-homomorphisms

$$
\operatorname{Hom}_{B}\left(P_{0} \otimes\left(\otimes_{j=1, \sum a_{j}=1}^{t} R_{\epsilon_{j}+2, a_{j}}\right) \oplus P_{1} \otimes\left(\otimes_{j=1}^{t} R_{\epsilon_{j}+2,0}\right), \mathbb{Z} / 2\right)
$$

when $m=\infty$ and $i$ lies in the range $i \geq 2 k-\alpha(k)$. This will show that $b \tilde{v}_{i} \hat{z}_{4 k} \neq 0$ from which $b^{e} \tilde{v}_{i} \hat{z}_{4 k} \neq 0$ for $e \geq 1$ follows because the isomorphism of $\S 2.5$ commutes with multiplication by $b$ when $s>0$.

I shall first give the argument to prove that $f$ does not exist and finally I shall explain where the $i \geq 2 k-\alpha(k)$ is necessary.

In degree $4 k+2 i+2$, suppose that we have the relation $\left(0, h_{i} \otimes g_{k}\right)=f \cdot d$. Then we shall apply $f \cdot d$ to all the $B$-basis elements in $P_{*} \otimes\left(\otimes_{j=1}^{t} R_{\left(\epsilon_{j}+2, *\right)}\right)$ in resolution degree 1 and homological degree $4 k+2 i+2$ and add the results in two ways to get a contradiction. The basis elements in question are

$$
\left\{\nu_{2 q+2} \otimes\left(\otimes_{j} \Sigma_{\epsilon_{j}+2,0,2 s_{j}}\right) \mid 2 q+2+\sum_{j} 2 s_{j}=4 k+2 i+2\right\}
$$

and (where $0 \leq a_{j} \leq 1$ and $\sum_{j} a_{j}=1$ )

$$
\left\{\sigma_{2 q+1} \otimes\left(\otimes_{j} \Sigma_{\epsilon_{j}+2, a_{j}, 2 s_{j}+a_{j}}\right) \mid 2 q+2+\sum_{j} 2 s_{j}+a_{j}=4 k+2 i+2\right\}
$$

disregarding, of course, the ones of this list which are zero by the convention introduced above. 
We have

$$
\begin{aligned}
& f\left(d\left(\nu_{2 q+2} \otimes\left(\otimes_{j} \Sigma_{\epsilon_{j}+2,0,2 s_{j}}\right)\right)\right) \\
& =f\left(S q^{1} \sigma_{2 q+1} \otimes\left(\otimes_{j} \Sigma_{\epsilon_{j}+2,0,2 s_{j}}\right)\right)+f\left(S q^{0,1} \sigma_{2 q-1} \otimes\left(\otimes_{j} \Sigma_{\epsilon_{j}+2,0,2 s_{j}}\right)\right)
\end{aligned}
$$

and (where $0 \leq a_{j} \leq 1$ and $\sum_{j} a_{j}=1$ )

$$
\begin{aligned}
& \quad f\left(d\left(\sigma_{2 q+1} \otimes\left(\otimes_{j} \Sigma_{\epsilon_{j}+2, a_{j}, 2 s_{j}+a_{j}}\right)\right)\right) \\
& =f\left(\sigma_{2 q+1} \otimes \ldots \otimes S q^{1} \Sigma_{\epsilon_{j}+2,0,2 s_{j}} \otimes \ldots\right) \\
& \quad+f\left(\sigma_{2 q+1} \otimes \ldots \otimes S q^{0,1} \Sigma_{\epsilon_{j}+2,0,2 s_{j}-2} \otimes \ldots\right)
\end{aligned}
$$

where in the last expression the $S q$ 's appear precisely in the unique factor for which $a_{j}$ was equal to one.

Now fix a $t+1$-tuple $\left(q, s_{1}, \ldots, s_{t}\right)$ such that $4 k+2 i+2=2 q+2+\sum_{j} 2 s_{j}$ and consider the sum

$$
\begin{aligned}
& f\left(S q^{1} \sigma_{2 q+1} \otimes\left(\otimes_{j} \Sigma_{\epsilon_{j}+2,0,2 s_{j}}\right)\right)+\sum_{j=1}^{t} f\left(\sigma_{2 q+1} \otimes \ldots \otimes S q^{1} \Sigma_{\epsilon_{j}+2,0,2 s_{j}} \otimes \ldots\right) \\
& =S q^{1}\left(f\left(\sigma_{2 q+1} \otimes\left(\otimes_{j} \Sigma_{\epsilon_{j}+2,0,2 s_{j}}\right)\right)\right) \\
& =0
\end{aligned}
$$

because $S q^{1}$ acts trivially on $\mathbb{Z} / 2$ for dimensional reasons. Similarly

$$
\begin{aligned}
& f\left(S q^{0,1} \sigma_{2 q-1} \otimes\left(\otimes_{j} \Sigma_{\epsilon_{j}+2,0,2 s_{j}}\right)\right) \\
& \quad+\sum_{j=1}^{t} f\left(\sigma_{2 q+1} \otimes \ldots \otimes S q^{0,1} \Sigma_{\epsilon_{j}+2,0,2 s_{j}} \otimes \ldots\right) \\
& =0 .
\end{aligned}
$$

Therefore applying $f \cdot d$ to each of the basis elements listed above and adding the results yields zero modulo 2 .

Now consider what happens if we apply $\left(0, h_{i} \otimes g_{k}\right)$ to each of the basis elements listed above and add the results. The sum equals 1 because the map

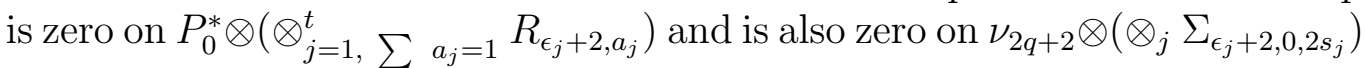
unless $q=i$ and $2 s_{j}=2^{\epsilon_{j}+2}$ for $j=1, \ldots, t$.

This contradiction completes the proof of part (iii) except that it remains to explain why we need the condition that

$$
i \geq 2^{\epsilon_{1}+1}+2^{\epsilon_{2}+1}+\ldots+2^{\epsilon_{t}+1}-t+1 .
$$

We require that $i$ be large enough so that all the elements $\nu_{2 q+2} \otimes\left(\otimes_{j} \Sigma_{\epsilon_{j}+2,0,2 s_{j}}\right)$ and $\sigma_{2 q+1} \otimes\left(\otimes_{j} \Sigma_{\epsilon_{j}+2, a_{j}, 2 s_{j}+a_{j}}\right)$ over which we want to sum are permissible within homological degree $2 i+2+4 k$. However if $i \geq 2^{\epsilon_{1}+1}+2^{\epsilon_{2}+1}+\ldots+$ $2^{\epsilon_{t}+1}-t+1$ then

$$
2 i+2+4 k \geq 2^{\epsilon_{1}+3}-2+2^{\epsilon_{2}+3}-2+\ldots+2^{\epsilon_{t}+3}-2+2 \geq \sum_{j=1}^{t} 2 s_{j}
$$


for all possible choices of the $s_{j}$ 's involved in the sum.

2.9. The maps $\iota_{k, l}$

As in $\S 2.5$ let $b u$ and bo denote the 2-localised, connective unitary and orthogonal K-theory spectra, respectively. Consider a left-bu-module spectrum map

$$
\iota: b u \wedge\left(F_{4 k} / F_{4 k-1}\right) \longrightarrow b u \wedge\left(F_{4 l} / F_{4 l-1}\right) .
$$

This map is determined up to homotopy by its restriction, via the unit of $b u$, to $\left(F_{4 k} / F_{4 k-1}\right)$. By S-duality this restriction is equivalent to a map of the form

$$
S^{0} \longrightarrow D\left(F_{4 k} / F_{4 k-1}\right) \wedge b u \wedge\left(F_{4 l} / F_{4 l-1}\right),
$$

which $D X$ denotes the S-dual of $X$. Maps of this form are studied by means of the (collapsed) Adams spectral sequence (see [20] §3.1)

$$
\begin{gathered}
E_{2}^{s, t}=\operatorname{Ext}_{B}^{s, t}\left(\tilde{H}^{*}\left(D\left(F_{4 k} / F_{4 k-1}\right) ; \mathbb{Z} / 2\right) \otimes \tilde{H}^{*}\left(F_{4 l} / F_{4 l-1} ; \mathbb{Z} / 2\right), \mathbb{Z} / 2\right) \\
\Longrightarrow \pi_{t-s}\left(D\left(F_{4 k} / F_{4 k-1}\right) \wedge\left(F_{4 l} / F_{4 l-1}\right) \wedge b u\right) \otimes \mathbb{Z}_{2}
\end{gathered}
$$

where $\mathbb{Z}_{2}$ denotes the 2 -adic integers. It is shown in [20] that such maps $\iota$ are trivial when $l<k$ and form a copy of the $\mathbb{Z}_{2}$ when $l \geq k$. Following [10] and [20] we choose left-bu-module spectrum maps

$$
\iota_{k, l}: \text { bu } \wedge\left(F_{4 k} / F_{4 k-1}\right) \longrightarrow b u \wedge\left(F_{4 l} / F_{4 l-1}\right)
$$

to satisfy $\iota_{k, k}=1, \iota_{k, l}=\iota_{l+1, l} \iota_{l+2, l+1} \ldots \iota_{k, k-1}$ for all $k-l \geq 2$ and each $\iota_{t+1, t}$ is a $\mathbb{Z}_{2}$-module generator of the group of such left-bu-module maps.

Let $\tilde{z}_{4 k} \in \pi_{4 k}\left(b u \wedge\left(F_{4 k} / F_{4 k-1}\right)\right) \otimes \mathbb{Z}_{2}$ denote the element represented by the smash product of the unit $\eta$ of the bu-spectrum with the inclusion of the bottom cell $j_{k}$ into $F_{4 k} / F_{4 k-1}$ (see [10] $\S 2.12$ )

$$
S^{0} \wedge S^{4 k} \stackrel{\eta \wedge j_{k}}{\longrightarrow} b u \wedge F_{4 k} / F_{4 k-1}
$$

and let $v_{2 i-1} \in \pi_{2 i-1}\left(b u \wedge \mathbb{R P}^{\infty}\right) \otimes \mathbb{Z}_{2}=b u_{2 i-1}\left(\mathbb{R P}^{\infty}\right)$ be as in Proposition 2.2. Then we have the exterior product

$$
v_{2 i-1} \tilde{z}_{4 k} \in \pi_{4 k+2 i-1}\left(\text { bu } \wedge \mathbb{R P}^{\infty} \wedge\left(F_{4 k} / F_{4 k-1}\right) \otimes \mathbb{Z}_{2}\right.
$$

which is non-zero and is represented by $\tilde{v}_{2 i-1} \hat{z}_{4 k}$ in the collapsed Adams spectral sequence whose $E_{2}$-term is described in $\S 2.5$.

The following formula is central to the proof in $\S 3.8$ of our main theorem.

\section{Proposition 2.10.}

For $l<k$, for some 2-adic unit $\mu_{4 k, 4 l}$,

$$
\left(\iota_{k, l}\right)_{*}\left(v_{2 i-1} \tilde{z}_{4 k}\right)=\mu_{4 k, 4 l} 2^{4 k-4 l-\alpha(k)+\alpha(l)} v_{2 i+4 k-4 l-1} \tilde{z}_{4 l}
$$




\section{Proof}

Since $\iota_{k, l}$ is a left- $b$-module map we have $\left(\iota_{k, l}\right)_{*}\left(v_{2 i-1} \tilde{z}_{4 k}\right)=v_{2 i-1}\left(\iota_{k, l}\right)_{*}\left(\tilde{z}_{4 k}\right)$ and, by ([10] Proposition 3.2) $\left(\iota_{k, l}\right)_{*}\left(\tilde{z}_{4 k}\right)=\mu_{4 k, 4 l} 2^{2 k-2 l-\alpha(k)+\alpha(l)} u^{2 k-2 l} \tilde{z}_{4 l}$ for some 2-adic unit $\mu_{4 k, 4 l}$. The result follows since, by Proposition 2.2,

$$
v_{2 i-1} \mu_{4 k, 4 l} l^{2 k-2 l-\alpha(k)+\alpha(l)} u^{2 k-2 l} \tilde{z}_{4 l}=v_{2 i+4 k-4 l-1} \mu_{4 k, 4 l} 2^{4 k-4 l-\alpha(k)+\alpha(l)} \tilde{z}_{4 l} .
$$

\section{Applichtions}

3.1. The main diagram

In this section we are going to apply the results of the previous section together with the upper triangular yoga of [20] and [10] to the following partially commutative diagram to prove Theorem 1.4. In the diagram $\eta$ is the unit of $b u, c$ is complexification, $\mu$ is the $b u$-multiplication and $\psi^{3}$ is the Adams operation. The homomorphism $\lambda_{*}$ is equal to $(\mu \wedge 1)_{*} \cdot(1 \wedge c \wedge 1)_{*}$.

The diagram does not commute because the right-hand oblique vertical rectangle does not commute. However the upper and lower triangles, the back rectangle and the front left-hand oblique vertical rectangle do commute. 


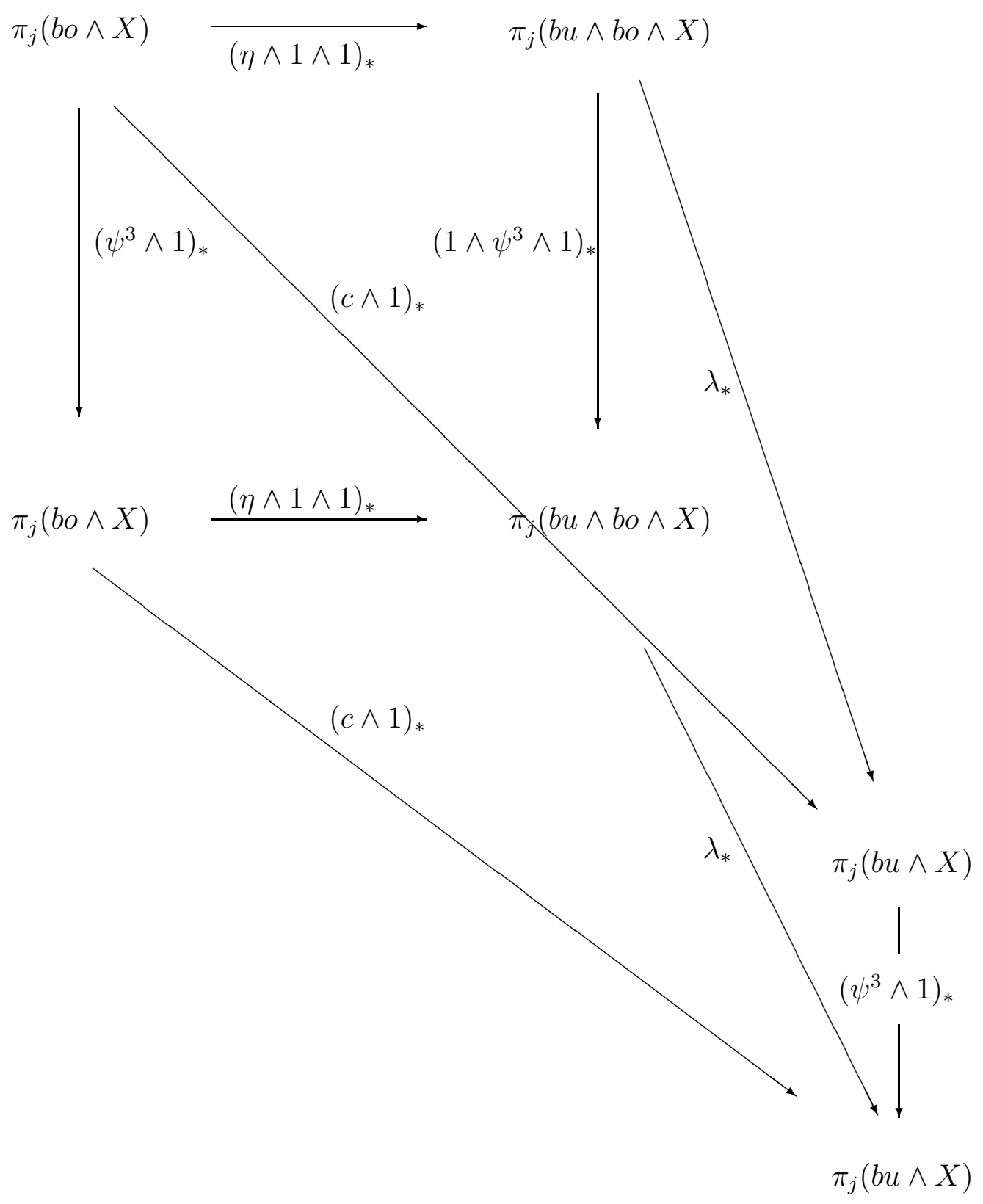

Example 3.2. $\quad \mathbb{R} \mathbb{P}^{8 m-1}$

Let $\tilde{K U}$ denote reduced 2-local periodic unitary K-theory and let $b u$, bo denote the associated connective K-theories. In this example, by way of illustration, we shall show how to use the results of [20] and [10] to calculate 
the map

$$
(\eta \wedge 1 \wedge 1)_{*}: b o_{8 m-1}\left(\mathbb{R} P^{8 m-1}\right) \longrightarrow \pi_{8 m-1}\left(b u \wedge \text { bo } \wedge \mathbb{R P}^{8 m-1}\right) .
$$

From $[5]$ we have $\tilde{K U} U^{0}\left(\mathbb{R P}^{2 t-1}\right) \cong \tilde{K} U^{0}\left(\mathbb{R P} P^{2 t-2}\right) \cong \mathbb{Z} / 2^{t-1}$ and $\tilde{K U}{ }^{1}\left(\mathbb{R P}^{2 t-1}\right) \cong \mathbb{Z}_{2}$. The $K U$-theory universal coefficient theorem (proved by the method of [6]) shows that

$$
\tilde{K} U_{1}\left(\mathbb{R} \mathbb{P}^{2 t-1}\right) \cong \mathbb{Z}_{2}\left\langle F_{2}\right\rangle \oplus \mathbb{Z} / 2^{t-1}\left\langle F_{1}\right\rangle, \tilde{K} U_{0}\left(\mathbb{R P}^{2 t-1}\right)=0 .
$$

The Adams operation $\psi^{3}$ gives a stable operation on 2-local $K U$-homology and in the book review [19] a (then) new, one-line proof of the non-existence of maps of Hopf invariant one based on the formula

$$
\psi^{3}\left(F_{2}\right)=\left(\left(3^{t}-1\right) / 2\right) F_{1}+3^{t} F_{2}, \psi^{3}\left(F_{1}\right)=F_{1} .
$$

The canonical map from $b u_{2 t-1}\left(\mathbb{R P}^{2 t-1}\right)$ to $\tilde{K} U_{2 t-1}\left(\mathbb{R} P^{2 t-1}\right)$ is an isomorphism commuting with $\psi^{3}$ so that

$$
b u_{2 t-1}\left(\mathbb{R P}^{2 t-1}\right) \cong \mathbb{Z}_{2}\left\langle F_{2}\right\rangle \oplus \mathbb{Z} / 2^{t-1}\left\langle F_{1}\right\rangle
$$

with the $\psi^{3}$ acting on the generators by the formulae

$$
\psi^{3}\left(F_{2}\right)=F_{2}+u_{t}\left(\left(3^{t}-1\right) / 2\right) F_{1}, \quad \psi^{3}\left(F_{1}\right)=F_{1},
$$

where $u_{t}$ is an odd integer. When $t=4 \mathrm{~m}$ the complexification map is an isomorphism giving, in the notation of Proposition 2.2,

$$
b o_{8 m-1}\left(\mathbb{R P}^{8 m-1}\right) \cong b u_{8 m-1}\left(\mathbb{R P}^{8 m-1}\right) \cong \mathbb{Z}_{2}\left\langle\iota_{8 m-1}\right\rangle \oplus \mathbb{Z} / 2^{4 m-1}\left\langle v_{8 m-3} u\right\rangle
$$

where the second summand is $b_{8 m-1}\left(\mathbb{R P}^{8 m-2}\right) \cong b u_{8 m-1}\left(\mathbb{R P}^{8 m-2}\right)$ and

$$
\psi^{3}\left(\iota_{8 m-1}\right)=\iota_{8 m-1}+u_{4 m}\left(\left(3^{4 m}-1\right) / 2\right) v_{8 m-3} u, \quad \psi^{3}\left(v_{8 m-3} u\right)=v_{8 m-3} u .
$$

Proposition 2.4, the discussion of $§ 2.5$ and Theorem 2.6 easily imply (c.f. [2] Lemma 17.12) that the Adams spectral sequence

$\operatorname{Ext}_{B}^{s, t}\left(\tilde{H}^{*}\left(\mathbb{R P}^{8 m-2} \wedge\left(F_{4 k} / F_{4 k-1}\right) ; \mathbb{Z} / 2\right), \mathbb{Z} / 2\right) \Longrightarrow b u_{t-s}\left(\mathbb{R P}^{8 m-2} \wedge\left(F_{4 k} / F_{4 k-1}\right)\right)$ collapses and that for $1 \leq k \leq 2 m-1$ and $4 m \geq 4 k-\alpha(k)+1$

$$
\begin{aligned}
b u_{8 m-1}\left(\mathbb{R P}^{8 m-1} \wedge\left(F_{4 k} / F_{4 k-1}\right)\right) & \cong b u_{8 m-1}\left(\mathbb{R P}^{8 m-2} \wedge\left(F_{4 k} / F_{4 k-1}\right)\right) \\
& \cong V_{k} \oplus \mathbb{Z} / 2^{4 m-4 k+\alpha(k)}\left\langle v_{8 m-4 k-1} \tilde{z}_{4 k}\right\rangle
\end{aligned}
$$

where $V_{k}$ is a finite-dimensional $\mathbb{F}_{2}$-vector space consisting of elements which are detected in mod 2 cohomology (i.e. in Adams filtration zero) in the spectral sequence. If $8 m-1 \leq 8 k-2 \alpha(k)+1$ then the group is a $\mathbb{Z} / 2$-vector space of the form

$$
b u_{8 m-1}\left(\mathbb{R} \mathbb{P}^{8 m-1} \wedge\left(F_{4 k} / F_{4 k-1}\right)\right) \cong V_{k} \oplus \mathbb{Z} / 2\left\langle v_{8 m-4 k-1} \tilde{z}_{4 k}\right\rangle
$$

entirely in Adams filtration zero and if $k \geq 2 m$ the group is zero.

By means of the 2-local equivalence $\hat{L}$ of $\S 2.5$ we have a direct sum decomposition

$$
\hat{L}_{*}: \oplus_{k \geq 0} b u_{*}\left(\mathbb{R} \mathbb{P}^{8 m-1} \wedge\left(F_{4 k} / F_{4 k-1}\right)\right) \stackrel{\cong}{\longrightarrow} \pi_{*}\left(b u \wedge b o \wedge \mathbb{R P}^{8 m-1}\right)
$$


and by means of this identification we may write the element $(\eta \wedge 1 \wedge 1)_{*}\left(\iota_{8 m-1}\right) \in \pi_{8 m-1}\left(b u \wedge b o \wedge \mathbb{R P}^{8 m-1}\right)$ as a vector $\left(w_{0}, w_{1}, \ldots, w_{2 m-1}\right)$ with $w_{k} \in b u_{8 m-1}\left(\mathbb{R P}^{8 m-1} \wedge\left(F_{4 k} / F_{4 k-1}\right)\right)$. In the diagram of $\S 3.1$ with $X=\mathbb{R P}^{8 m-1}$ and $j=8 m-1$ the map $(c \wedge 1)_{*}$ is an isomorphism which sends $\iota_{8 m-1}$ to itself. Hence $w_{0}=\iota_{8 m-1}$.

According to the main theorem of [20] a left-bu-module self-equivalence of $b u \wedge b o$ inducing the identity on mod 2 homology determines a unique conjugacy class in the upper triangular group with entries in the 2-adic integers. According to the main theorem of [10] the conjugacy class associated to the map $1 \wedge \psi^{3}$ is equal to

$$
\left(\begin{array}{cccccc}
1 & 1 & 0 & 0 & 0 & \ldots \\
0 & 9 & 1 & 0 & 0 & \ldots \\
0 & 0 & 9^{2} & 1 & 0 & \ldots \\
0 & 0 & 0 & 9^{3} & 1 & \ldots \\
\vdots & \vdots & \vdots & \vdots & \vdots & \vdots
\end{array}\right) .
$$

In practical terms this means that, for any positive integer $N$, we may choose $\hat{L}$ in $\S 2.5$ so that, for all $k \leq N, 1 \wedge \psi^{3}$ maps the wedge summand bu $\wedge\left(F_{4 k} / F_{4 k-1}\right)$ to itself by $9^{k}$ times the identity map, to bu $\wedge\left(F_{4 k-4} / F_{4 k-5}\right)$ by $\iota_{k, k-1}$ and to all other wedge summands $b u \wedge\left(F_{4 t} / F_{4 t-1}\right)$ trivially if $t \leq N$. If we choose $\hat{L}$ in this manner, taking $N$ very much larger than $8 m-1$, we have

$$
\begin{aligned}
& \left(1 \wedge \psi^{3} \wedge 1\right)_{*}\left((\eta \wedge 1 \wedge 1)_{*}\left(\iota_{8 m-1}\right)\right) \\
& =\left(1 \wedge \psi^{3} \wedge 1\right)_{*}\left(\iota_{8 m-1}, w_{1}, w_{2}, \ldots, w_{2 m-1}\right) \\
& =\left(\iota_{8 m-1}+\left(\iota_{1,0}\right)_{*}\left(w_{1}\right), 9 w_{1}+\left(\iota_{2,1}\right)_{*}\left(w_{2}\right), 9^{2} w_{2}+\left(\iota_{3,2}\right)_{*}\left(w_{3}\right), \ldots, 9^{2 m-1} w_{2 m-1}\right) .
\end{aligned}
$$

On the other hand this element is equal to

$$
\begin{aligned}
& (\eta \wedge 1 \wedge 1)_{*}\left(\left(\psi^{3} \wedge 1\right)_{*}\left(\iota_{8 m-1}\right)\right) \\
& =(\eta \wedge 1 \wedge 1)_{*}\left(\iota_{8 m-1}+u_{4 m}\left(\left(3^{4 m}-1\right) / 2\right) v_{8 m-3} u\right) \\
& =\left(\iota, w_{1}, w_{2}, \ldots, w_{2 m-1}\right)+u_{4 m}\left(\left(3^{4 m}-1\right) / 2\right)\left(v_{8 m-3} u, \tilde{w}_{1}, \tilde{w}_{2}, \ldots, \tilde{w}_{2 m-1}\right)
\end{aligned}
$$

where $(\eta \wedge 1 \wedge 1)_{*}\left(v_{8 m-3} u\right)=\left(v_{8 m-3} u, \tilde{w}_{1}, \tilde{w}_{2}, \ldots, \tilde{w}_{2 m-1}\right)$. 
Equating coordinates we obtain a string of equations

$$
\begin{aligned}
& \left(\iota_{1,0}\right)_{*}\left(w_{1}\right)=u_{4 m}\left(\left(3^{4 m}-1\right) / 2\right) v_{8 m-3} u \in b u_{8 m-1}\left(\mathbb{R P}^{8 m-1}\right) \\
& (9-1) w_{1}+\left(\iota_{2,1}\right)_{*}\left(w_{2}\right) \\
& =u_{4 m}\left(\left(3^{4 m}-1\right) / 2\right) \tilde{w}_{1} \in b u_{8 m-1}\left(b u \wedge \mathbb{R P}^{8 m-2} \wedge\left(F_{4} / F_{3}\right)\right), \\
& \left(9^{2}-1\right) w_{2}+\left(\iota_{3,2}\right)_{*}\left(w_{3}\right) \\
& =u_{4 m}\left(\left(3^{4 m}-1\right) / 2\right) \tilde{w}_{2} \in b u_{8 m-1}\left(b u \wedge \mathbb{R P}^{8 m-2} \wedge\left(F_{8} / F_{7}\right)\right), \\
& \quad \vdots \quad \vdots \\
& \left(9^{k}-1\right) w_{k}+\left(\iota_{k+1, k}\right)_{*}\left(w_{k+1}\right) \\
& =u_{4 m}\left(\left(3^{4 m}-1\right) / 2\right) \tilde{w}_{k} \in b u_{8 m-1}\left(\mathbb{R P}^{8 m-2} \wedge\left(F_{4 k} / F_{4 k-1}\right)\right), \\
& \vdots \\
& \quad \vdots \\
& \vdots
\end{aligned}
$$

There is a relation between $w_{i}$ and $\tilde{w}_{i}$ of the form $2 w_{i}=r_{8 m-1} \tilde{w}_{i}$ for all $i$ where $r_{8 m-1}$ is an odd integer. For we have a cofibration

$$
S^{8 m-1} \stackrel{\pi}{\longrightarrow} \mathbb{R P}^{8 m-1} \stackrel{\phi}{\longrightarrow} \mathbb{R P}^{8 m}
$$

in which $\pi$ is the canonical projection. Also $\phi_{*}\left(\iota_{8 m-1}\right)$ generates $b u_{8 m-1}\left(\mathbb{R P}^{8 m}\right)$ so, by Proposition 2.2, $\phi_{*}\left(2 \iota_{8 m-1}-r_{8 m-1} v_{8 m-3} u\right)=0$ for some odd integer $r_{8 m-1}$. Therefore $2 \iota_{8 m-1}-r_{8 m-1} v_{8 m-3} u$ originates in $b u_{8 m-1}\left(S^{8 m-1}\right)$ and

$$
(1 \wedge \eta \wedge 1)_{*}\left(2 \iota_{8 m-1}\right)=\left(2 \iota_{8 m-1}-r_{8 m-1} v_{8 m-3} u, 0,0,0, \ldots\right) .
$$

If we write $u_{4 m}^{\prime}$ for the 2 -adic unit $u_{4 m} r_{8 m-1}^{-1}$ our string of equations simplifies to

$$
\begin{gathered}
\left(\iota_{1,0}\right)_{*}\left(w_{1}\right)=u_{4 m}\left(\left(3^{4 m}-1\right) / 2\right) v_{8 m-3} u \in b u_{8 m-1}\left(\mathbb{R P} P^{8 m-1}\right), \\
(9-1) w_{1}+\left(\iota_{2,1}\right)_{*}\left(w_{2}\right)=2 u_{4 m}^{\prime}\left(\left(3^{4 m}-1\right) / 2\right) w_{1}, \\
\left(9^{2}-1\right) w_{2}+\left(\iota_{3,2}\right)_{*}\left(w_{3}\right)=2 u_{4 m}^{\prime}\left(\left(3^{4 m}-1\right) / 2\right) w_{2}, \\
\vdots \quad \vdots \quad \vdots \quad \vdots \\
\left(9^{k}-1\right) w_{k}+\left(\iota_{k+1, k}\right)_{*}\left(w_{k+1}\right)=2 u_{4 m}^{\prime}\left(\left(3^{4 m}-1\right) / 2\right) w_{k}, \\
\vdots \quad \vdots \quad \\
\vdots \\
\quad
\end{gathered}
$$


We conclude the discussion of this example with the following result concerning the homomorphism $\left(\iota_{k, k-1}\right)_{*}$

$b u_{8 m-1}\left(\mathbb{R P}^{8 m-2} \wedge\left(F_{4 k} / F_{4 k-1}\right)\right) \otimes \mathbb{Z}_{2} \longrightarrow b u_{8 m-1}\left(\mathbb{R P}^{8 m-2} \wedge\left(F_{4 k-4} / F_{4 k-5}\right)\right) \otimes \mathbb{Z}_{2}$.

\section{Proposition 3.3.}

In the notation of Proposition 2.10 and Example 3.2

(i) $\left(\iota_{1,0}\right)_{*}\left(v_{8 m-5} \tilde{z}_{4}\right)=\mu_{4,0} 2^{2} v_{8 m-3} u$,

(ii) If $2 \leq k \leq 2 m-1$ and $4 m \geq 4 k-\alpha(k)+1$ then $\left(\iota_{k, k-1}\right)_{*}$

$V_{k} \oplus \mathbb{Z} / 2^{4 m-4 k+\alpha(k)}\left\langle v_{8 m-4 k-1} \tilde{z}_{4 k}\right\rangle \longrightarrow V_{k-1} \oplus \mathbb{Z} / 2^{4 m-4 k+4+\alpha(k-1)}\left\langle v_{8 m-4 k+3} \tilde{z}_{4 k-4}\right\rangle$

satisfies $\left(\iota_{k, k-1}\right)_{*}\left(v_{8 m-4 k-1} \tilde{z}_{4 k}\right)=\mu_{4 k, 4 k-4} 2^{4-\alpha(k)+\alpha(k-1)} v_{8 m-4 k+3} \tilde{z}_{4 k-4}$ where $\mu_{4 k, 4 k-4}$ is a 2-adic unit.

In particular, $\left(\iota_{k, k-1}\right)_{*}$ is injective on $\mathbb{Z} / 2^{4 m-4 k+\alpha(k)}\left\langle v_{8 m-4 k-1} \tilde{z}_{4 k}\right\rangle$ in cases (i) and (ii).

\section{Proof}

These formulae follow from those of Proposition 2.10, concerning $\mathbb{R P}^{\infty}$ together with the injectivity of the map from $b u_{8 m-1}\left(\mathbb{R P}^{8 m-2} \wedge\left(F_{4 k} / F_{4 k-1}\right)\right)$ to $b u_{8 m-1}\left(\mathbb{R P}^{\infty} \wedge\left(F_{4 k} / F_{4 k-1}\right)\right)$, which follows from the Adams spectral sequence via Proposition 2.4 and $\S 2.5$. The formulae make sense because $\alpha(k-1)=$ $\alpha(k)-1+\nu_{2}(k)$.

Example 3.4. The maps $\Theta_{2 j}$

In this example we shall study stable homotopy classes of maps of the form $\Theta_{2 j}: S^{2 j} \longrightarrow \mathbb{R P}^{2 j}$ with mapping cone $C\left(\Theta_{2 j}\right)$ such that, on 2-local connective K-theory

$$
b u_{2 j+1}\left(C\left(\Theta_{2 j}\right)\right) \cong \mathbb{Z}_{2}\left\langle\iota_{2 j+1}\right\rangle \oplus \mathbb{Z} / 2^{j}\left\langle v_{2 j-1} u\right\rangle
$$

for some 2-adic unit $u_{j}$

$$
\left(\psi^{3} \wedge 1\right)_{*}\left(\iota_{2 j+1}\right)=\iota_{2 j+1}+u_{j}\left(\left(3^{j}-1\right) / 4\right) v_{2 j-1} u .
$$

In other words, the $\psi^{3}$ e-invariant (see [1]) of $C\left(\Theta_{2 j}\right)$ is half that of $\mathbb{R P}^{2 j+1}$ in Example 3.2, which is the mapping cone of $\theta$ in the canonical cofibre sequence

$$
\mathbb{R P}^{2 j} \longrightarrow \mathbb{R P}^{2 j+1} \longrightarrow S^{2 j+1} \stackrel{\theta}{\longrightarrow} \Sigma \mathbb{R P}^{2 j} \text {. }
$$

For simplicity we shall restrict ourselves to the case when $j=4 m-1$. In this case there are isomorphisms of 2-local K-groups

$$
b_{8 m-1}\left(C\left(\Theta_{8 m-2}\right)\right) \cong b u_{8 m-1}\left(C\left(\Theta_{8 m-2}\right)\right) \cong b u_{8 m-1}\left(\mathbb{R P}^{8 m-1}\right)
$$

and for each $k \geq 1$

$$
b u_{8 m-1}\left(C\left(\Theta_{8 m-2}\right) \wedge\left(F_{4 k} / F_{4 k-1}\right)\right) \cong b u_{8 m-1}\left(\mathbb{R P}^{8 m-2} \wedge\left(F_{4 k} / F_{4 k-1}\right)\right) .
$$


Furthermore if $(\eta \wedge 1 \wedge 1)_{*}\left(\iota_{8 m-1}\right)=\left(\iota_{8 m-1}, w_{1}^{\prime}, w_{2}^{\prime}, \ldots, w_{2 m-1}^{\prime}\right)$ the e-invariant condition yields, as in Example 3.2, a string of equations

$$
\begin{aligned}
& \left(\iota_{1,0}\right)_{*}\left(w_{1}^{\prime}\right)=u_{4 m}^{\prime \prime}\left(\left(3^{4 m}-1\right) / 4\right) v_{8 m-3} u \in b u_{8 m-1}\left(\mathbb{R P}^{8 m-1}\right) \\
& (9-1) w_{1}^{\prime}+\left(\iota_{2,1}\right)_{*}\left(w_{2}^{\prime}\right) \\
& =u_{4 m}^{\prime \prime}\left(\left(3^{4 m}-1\right) / 4\right) \tilde{w}_{1} \in b u_{8 m-1}\left(b u \wedge \mathbb{R P}^{8 m-2} \wedge\left(F_{4} / F_{3}\right)\right), \\
& \left(9^{2}-1\right) w_{2}^{\prime}+\left(\iota_{3,2}\right)_{*}\left(w_{3}^{\prime}\right) \\
& =u_{4 m}^{\prime \prime}\left(\left(3^{4 m}-1\right) / 4\right) \tilde{w}_{2} \in b u_{8 m-1}\left(b u \wedge \mathbb{R P}^{8 m-2} \wedge\left(F_{8} / F_{7}\right)\right), \\
& \quad \vdots \\
& \vdots \\
& \left(9^{k}-1\right) w_{k}^{\prime}+\left(\iota_{k+1, k}\right)_{*}\left(w_{k+1}^{\prime}\right) \\
& =u_{4 m}^{\prime \prime}\left(\left(3^{4 m}-1\right) / 4\right) \tilde{w}_{k} \in b u_{8 m-1}\left(\mathbb{R P}^{8 m-2} \wedge\left(F_{4 k} / F_{4 k-1}\right)\right)
\end{aligned}
$$

where $u_{4 m}^{\prime \prime}$ is a 2-adic unit and the $\tilde{w}_{k}$ 's are the same as in Example 3.2.

Suppose that we have an element

$$
w \in V_{i} \oplus \mathbb{Z} / 2^{4 m-4 i+\alpha(i)}\left\langle v_{8 m-4 i-1} \tilde{z}_{4 i}\right\rangle
$$

we shall write $w \simeq 2^{N}$ if $w=\left(x, 2^{N}(2 t+1) v_{8 m-4 i-1} \tilde{z}_{4 i}\right)$ for some integers $t$ and $N<4 m-4 i+\alpha(i)$.

Also we observe that in Proposition 3.3(i) or (ii) we may choose $V_{k}$ so that $\left(\iota_{k, k-1}\right)_{*}\left(V_{k}\right)=0$. Choosing $V_{k}$ in this manner will simplify our subsequent calculations.

\section{Proposition 3.5.}

Let $m=(2 p+1) 2^{q}$ with $p \geq 1$. In the notation of Examples 3.2 and 3.4

(i) For $2 \leq k \leq 2^{q+1}, \iota_{k, k-1}\left(w_{k}\right) \simeq 2^{4+q}$ and $\iota_{k, k-1}\left(w_{k}^{\prime}\right) \simeq 2^{3+q}$.

(ii) $w_{2^{q+1}} \simeq 1$.

(iii) Under these hypotheses $\Theta_{8 m-2}$ does not exist.

\section{Proof}

First we observe that $k \leq 2^{q+1}$ implies that $4 m=p 2^{q+3}+2^{q+2} \geq 4 k-\alpha(k)+$ 1 so that we may apply Proposition 3.3. Therefore the relations $\left(\iota_{1,0}\right)_{*}\left(w_{1}^{\prime}\right)=$ $u_{4 m}^{\prime \prime}\left(\left(3^{4 m}-1\right) / 4\right) v_{8 m-3} u$ and $\nu_{2}\left(\left(9^{2 m}-1\right) / 4\right)=3+\nu_{2}(2 m)-2=2+q$ implies $w_{1}^{\prime} \simeq 2^{q}$ and similarly $w_{1} \simeq 2^{1+q}$ (once we observe that the condition $1+q<4 m-4+\alpha(1)$ is fulfilled). The relation

$$
(9-1) w_{1}+\left(\iota_{2,1}\right)_{*}\left(w_{2}\right)=u_{4 m}\left(\left(3^{4 m}-1\right) / 2\right) \tilde{w}_{1} \in V_{1} \oplus \mathbb{Z} / 2^{4 m-3}\left\langle v_{8 m-5} \tilde{z}_{4}\right\rangle
$$


implies that $\left(\iota_{2,1}\right)_{*}\left(w_{2}\right) \simeq 2^{4+q}$ and similarly $\left(\iota_{2,1}\right)_{*}\left(w_{2}^{\prime}\right) \simeq 2^{3+q}$, which starts an induction on $k$.

Suppose $2 \leq k<2^{q+1}$ that

$$
\iota_{k, k-1}\left(w_{k}\right) \simeq 2^{4+q} \in \mathbb{Z} / 2^{4 m-4 k+4+\alpha(k-1)} .
$$

Therefore $w_{k} \in \mathbb{Z} / 2^{4 m-4 k+\alpha(k)}$ satisfies $w_{k} \simeq 2^{\alpha(k)-\alpha(k-1)+q}=2^{q+1-\nu_{2}(k)}$. Then, since $\nu_{2}\left(9^{k}-1\right)=3+\nu_{2}(k)$,

$$
\left(9^{k}-1\right) w_{k}+\iota_{k+1, k}\left(w_{k+1}\right)=\left(9^{2 m}-1\right) w_{k}
$$

implies that $\iota_{k+1, k}\left(w_{k+1}\right) \simeq 2^{4+q}$, as required. Similarly $\iota_{k+1, k}\left(w_{k+1}^{\prime}\right) \simeq 2^{3+q}$.

Since $\left(\iota_{2^{q+1}, 2^{q+1}-1}\right)_{*}\left(v_{8 m-2^{q+3}-1} \tilde{z}_{2^{q+3}}\right)=\mu_{2^{q+3}, 2^{q+3}-4} 2^{q+4} v_{8 m-2^{q+3}+3} \tilde{z}_{2^{q+3}-4}$ we see that $w_{2^{q+1}} \simeq 1$ and that $w_{2^{q+1}}^{\prime}$ cannot exist.

\section{Proposition 3.6.}

Let $m=2^{q}$. In the notation of Examples 3.2 and 3.4

(i) For $2 \leq k \leq 2^{q}, \quad\left(\iota_{k, k-1}\right)_{*}\left(w_{k}\right) \simeq 2^{4+q}$ and $\left(\iota_{k, k-1}\right)_{*}\left(w_{k}^{\prime}\right) \simeq 2^{3+q}$.

(ii) In $\mathbb{Z} / 2 \oplus V_{2^{q}}, \quad w_{2^{q}}^{\prime} \simeq 1$.

\section{Proof}

This time we observe that $k \leq 2^{q}$ implies $4 m=2^{q+2} \geq 4 k-\alpha(k)+1$ so that Proposition 3.3 applies and therefore part (i) follows as in Proposition 3.5. For part (ii) we have

$$
\left(\iota_{2^{q}, 2^{q}-1}\right)_{*}\left(v_{8 m-2^{q+2}-1} \tilde{z}_{2^{q+2}}\right)=\mu_{2^{q+2}, 2^{q+2}-4} 2^{3+q} v_{8 m-2^{q+2}+3} \tilde{z}_{2^{q+2}-4}
$$

and the result follows.

Remark 3.7. (i) In Proposition $3.6\left(\iota_{2^{q}, 2^{q}-1}\right)_{*}$ has the form

$$
\mathbb{Z} / 2 \oplus V_{2^{q}} \longrightarrow \mathbb{Z} / 2^{q+4} \oplus V_{2^{q}-1}
$$

so that the first component of $\left(\iota_{2^{q}, 2^{q}-1}\right)_{*}\left(w_{2^{q}}\right)$ is zero and so is that of $w_{2^{q}}$.

(ii) When $m=2^{q}$ we have shown that $w_{k}^{\prime} \simeq 2^{q-\nu_{2}(k)}$ for $1 \leq k \leq 2^{q}$.

3.8. Proof of Theorem 1.4

Let $m=2^{q}$ then we have $\iota_{8 m-1} \in b o_{8 m-1}\left(C\left(\Theta_{8 m-2}\right)\right)$ giving a stable homotopy class $\iota_{8 m-1}: S^{2^{q+3}-1} \longrightarrow b o \wedge C\left(\Theta_{8 m-2}\right)$. Let $\iota: b u \longrightarrow H \mathbb{Z} / 2$ be the canonical cohomology class. Then, if $h_{8 m-1} \in H_{2^{q+3}-1}\left(\right.$ bo $\left.\wedge C\left(\Theta_{8 m-2}\right) ; \mathbb{Z} / 2\right)$ is the mod 2 Hurewicz image of $\iota_{8 m-1}$, it is represented by either of the compositions

$$
S^{2^{q+3}-1} \stackrel{\iota 8 m-1}{\longrightarrow} b o \wedge C\left(\Theta_{8 m-2}\right) \stackrel{(\eta \wedge 1 \wedge 1)}{\longrightarrow} b u \wedge b o \wedge C\left(\Theta_{8 m-2}\right) \stackrel{(\iota \wedge 1 \wedge 1)}{\longrightarrow} H \mathbb{Z} / 2 \wedge b o \wedge C\left(\Theta_{8 m-2}\right)
$$

or

$$
S^{2^{q+3}-1} \stackrel{\iota 8 m-1}{\longrightarrow} S^{0} \wedge b o \wedge C\left(\Theta_{8 m-2}\right) \stackrel{(\tilde{\eta} \wedge 1 \wedge 1)}{\longrightarrow} H \mathbb{Z} / 2 \wedge b o \wedge C\left(\Theta_{8 m-2}\right)
$$

where $\tilde{\eta}$ is the unit for $H \mathbb{Z} / 2$.

We have an isomorphism of $\mathbb{Z} / 2$-vector spaces

$$
H_{*}(b o ; \mathbb{Z} / 2) \cong \underset{k \geq 0}{\oplus_{18}} H_{*}\left(F_{4 k} / F_{4 k-1} ; \mathbb{Z} / 2\right)
$$


and

$$
H_{j}\left(C\left(\Theta_{8 m-2}\right) ; \mathbb{Z} / 2\right) \cong \begin{cases}\mathbb{Z} / 2\left\langle\iota_{8 m-1}\right\rangle & \text { if } j=8 m-1, \\ \mathbb{Z} / 2\left\langle v_{j}\right\rangle & \text { if } 1 \leq j=8 m-2, \\ 0 & \text { otherwise. }\end{cases}
$$

If the e-invariant is correct then we have shown that

$$
h_{8 m-1} \in H_{*}(b o ; \mathbb{Z} / 2) \otimes H_{*}\left(C\left(\Theta_{8 m-2}\right) ; \mathbb{Z} / 2\right)
$$

has the form

$$
h_{8 m-1}=\tilde{z}_{0} \otimes \iota_{2^{q+3}-1}+\tilde{z}_{2^{q+2}} \otimes v_{2^{q+2}-1}+\sum_{j=1}^{2^{q+2}-2} x_{8 m-1-j} \otimes v_{j}
$$

with $x_{8 m-1-j} \in H_{*}\left(F_{2^{q+2}+t} / F_{2^{q+2}-1+t} ; \mathbb{Z} / 2\right)$ with $t \geq 0$.

If $X \in \mathcal{A}^{2^{q+2}}$ is an element of the mod 2 Steenrod algebra of degree $2^{q+2}$ we write $X_{*}$ for the dual homomorphism on mod 2 homology, which decreases dimensions by $2^{q+2}$. Since $H^{*}(b o ; \mathbb{Z} / 2)$ is a cyclic $\mathcal{A}$-module generated by 1 in dimension zero there exists an $X$ such that $X_{*}\left(\tilde{z}_{2^{q+2}}\right)=\tilde{z}_{0}$. In fact, since the Hurewicz image of the bottom cell in $H_{4 k}\left(F_{4 k} / F_{4 k-1} ; \mathbb{Z} / 2\right)$ equals $\xi_{1}^{4 k}$, unravelling the homology isomorphism between $H_{*}(b u ; \mathbb{Z} / 2) \otimes H_{*}(b o ; \mathbb{Z} / 2)$ and $H_{*}(b u ; \mathbb{Z} / 2) \otimes H_{*}\left(\wedge_{l \geq 0} F_{4 l} / F_{4 l-1} ; \mathbb{Z} / 2\right)$ described in [20] one can show that $S q_{*}^{4 k}\left(\tilde{z}_{4 k}\right)=\tilde{z}_{0}$.

Since $h_{8 m-1}$ is stably spherical we have $0=S q_{*}^{2^{q+2}}\left(h_{8 m-1}\right)$ so that

$$
\begin{aligned}
& \tilde{z}_{0} \otimes S q_{*}^{2^{q+2}}\left(\iota_{2^{q+3}-1}\right) \\
& =\tilde{z}_{0} \otimes v_{2^{q+2}-1}+\tilde{z}_{2^{q+2}} \otimes S q_{*}^{2^{q+2}}\left(v_{2^{q+2}-1}\right) \\
& \quad+\sum_{j=1}^{2^{q+2}-1} S q_{*}^{j}\left(\tilde{z}_{2^{q+2}}\right) \otimes S q_{*}^{2^{q+2}-j}\left(v_{2^{q+2}-1}\right)+\sum_{j=1}^{2^{q+2}-2} x_{8 m-1-j} \otimes S q_{*}^{2^{q+2}}\left(v_{j}\right) \\
& \quad+\sum_{a=1}^{2^{q+2}-1} \sum_{j=1}^{2^{q+2}-2} S q_{*}^{a}\left(x_{8 m-1-j}\right) \otimes S q_{*}^{2^{q+2}-a}\left(v_{j}\right)
\end{aligned}
$$

which implies, comparing coefficients of $\tilde{z}_{0}$, that $S q_{*}^{2^{q+2}}\left(\iota_{2^{q+3}-1}\right)=v_{2^{q+2}-1}$ so that $\iota_{8 m-1}$ is detected by $S q^{2^{q+2}}$ on its mapping cone, as required. That is:-

$$
\begin{aligned}
& H_{2^{q+3}-1}\left(C\left(\Theta_{2^{q+3}-2}\right) ; \mathbb{Z} / 2\right) \stackrel{\cong}{\longrightarrow} H_{2^{q+3}-1}\left(S^{2^{q+3}-1} ; \mathbb{Z} / 2\right), \\
& S q_{*}^{2^{q+2}}: H_{2^{q+3}-1}\left(C\left(\Theta_{2^{q+3}-2}\right) ; \mathbb{Z} / 2\right) \stackrel{\cong}{\longrightarrow} H_{2^{q+2}-1}\left(C\left(\theta_{2^{q+3}-2}\right) ; \mathbb{Z} / 2\right), \\
& H_{2^{q+2}-1}\left(\Sigma \mathbb{R P}^{2^{q+3}-2} ; \mathbb{Z} / 2\right) \stackrel{\cong}{\longrightarrow} H_{2^{q+2}-1}\left(C\left(\Theta_{2^{q+3}-2}\right) ; \mathbb{Z} / 2\right) .
\end{aligned}
$$

Conversely, if this detection by $S q^{2^{q+2}}$ is correct then $w_{2^{q}}^{\prime} \simeq 1$ and therefore the e-invariant is right.

When $m$ is not a power of 2, Proposition 3.5(iii) shows that $\Theta_{8 m-2}$ with this e-invariant cannot exist. For mod 2 cohomology one can appeal to a theorem 
of Browder [8] or alternatively well-known formulae for the $\mathcal{A}$-module action on the mod 2 cohomology of real projective space show that $\Theta_{8 m-2}$ cannot be detected by a primary operation on $H^{*}\left(C\left(\Theta_{8 m-2}\right) ; \mathbb{Z} / 2\right)$.

Department of Pure Mathematics, University of Sheffield, Sheffield S3 7RH, England.

v.snaith@sheffield.ac.uk

\section{REFERENCES}

[1] J.F. Adams: J(X) I-IV; Topology 2 (1963) 181-195, Topology 3 (1964) 137-171, Topology 3 (1964) 193-222, Topology 5 (1966) 21-71.

[2] J.F. Adams: Stable Homotopy and Generalised Homology; University of Chicago Press (1974).

[3] J.F. Adams: The Kahn-Priddy Theorem; Proc. Camb. Phil. Soc. (1973) 45-55.

[4] J.F. Adams and H.R. Margolis: Modules over the Steenrod algebra; Topology 10 (1971) 271-282.

[5] M.F. Atiyah: K-Theory; Benjamin, New York (1967).

[6] M.F. Atiyah: Vector bundles and the Künneth formula; Topology 1 (1962) 245-248.

[7] M.G. Barratt, J.D.S. Jones and M. Mahowald: The Kervaire invariant and the Hopf invariant; Proc. Conf. Algebraic Topology Seattle Lecture Notes in Math. \#1286 Springer Verlag (1987).

[8] W. Browder: The Kervaire invariant of framed manifolds and its generalizations; Annals of Math. (2) 90 (1969) 157-186.

[9] E.H. Brown and F.P. Peterson: On the stable decomposition of $\Omega^{2} S^{r+2}$; Trans. Amer. Math. Soc. 243 (1978) 287-298.

[10] J. Barker and V.P. Snaith: $\psi^{3}$ as an upper triangular matrix; K-theory 36 (2005) 91-114.

[11] J.D.S. Jones and E.G. Rees: Kervaire's invariant for framed manifolds; Algebraic and geometric topology, Proc. Symp. Pure Math XXXII (Part 2) Amer. Math. Soc. (1978) 111-117.

[12] D. S. Kahn and S. B. Priddy: The transfer and stable homotopy theory; Proc. Camb. Phil. Soc. 83 (1978) 103-112 (See also: Applications of the transfer to stable homotopy theory; Bull. A.M.Soc. 741 (1972) 981-987).

[13] J. Klippenstein and V.P. Snaith: A conjecture of Barratt-Jones-Mahowald concerning framed manifolds having Kervaire invariant one; Topology (4) 27 (1988) 387-392.

[14] K. Knapp: $\operatorname{Im}(J)$-theory and the Kervaire invariant; Math. Zeit. 226 (1997) 103-125.

[15] S.O. Kochman: Stable Homotopy Groups of Spheres; Lecture Notes in Math. \#1423, Springer-Verlag (1990).

[16] R.J. Milgram: Symmetries and operations in homotopy theory; Proc. Symp. Pure Math XXII Amer. Math. Soc. (1971) 203-210.

[17] V.P. Snaith: A stable decomposition of $\Omega^{n} S^{n} X$; J. London Math. Soc. 2 (1974) 577-583.

[18] V.P. Snaith and J. Tornehave, On $\pi_{*}(B O)$ and the Arf invariant of framed manifolds; Contemporary Mathematics 12 (1989) 299-313.

[19] V.P. Snaith: Review of Some applications of topological K-theory by N. Mahammed, R. Piccinini and U. Suter; Bull.A.M.Soc. v.8 (1) 117-120 (1983).

[20] V.P. Snaith: The upper triangular group and operations in algebraic K-theory; Topology 41 (2002) 1259-1275.

[21] V.P. Snaith: Hurewicz images in BP and the Arf-Kervaire invariant; Glasgow J.Math. 44 (2002) 9-27. 
[22] N.E. Steenrod: Cohomology operations; Annals Math. Studies \#50 (written and revised by D.B.A. Epstein) Princeton Univ. Press (1962). 